\title{
Simulación de vuelco con ansys y ls-dyna de una superestructura de un autobús y su incidencia en el área de supervivencia.
}

\author{
Simulation of overturning with ansys and ls-dyna of a superstructure of a bus \\ and its incidence in the area of survival.
}

\author{
Cesar Hernán Arroba Arroba. ${ }^{1}$, Manolo Alexander Córdova Suárez. ${ }^{2}$, Cristian German \\ Santiana Espín. ${ }^{3}$, Gustavo Efraín Carrera Oña. ${ }^{4}$
}

Recibido: 12-09-2019 / Revisado: 06-10-209 /Aceptado: 07-11-2019/ Publicado: 05-12-2019

\begin{abstract}
DOI:

https://doi.org/10.32/cienciadigital.v3i1.947

Survival space was studied in a section of the superstructure of the Interprovincial Service Bus manufactured by Miral Autobuses through software, Ansys and Ls-Dyna undergoing a rollover test. First, the material used by Miral Autobuses was characterized in the manufacture of the bus superstructure, determining mechanical properties that are necessary for the LsDyna software. Validation of results issued by Ls-Dyna was performed simulating the tensile, flexural, and flexural tests of a section of the gantry and comparing with the results of physical tests performed on the universal machine.

Methods: The study begins with the identification of the materials required by the manufacturer according to the norm Ecuadorian Technical Standard NTE INEN 1323: 2009 first revision "Automotive Vehicles. Bus Body Requirements"and then the rollover simulation was carried out with the Ansys and Ls-Dyna software of a section of the bus superstructure to determine if the survival space is invaded, finding improvement opportunities and subsequently performing a redesign of the superstructure and comply with Regulation No. 66 of the United Nations Economic Commission for Europe for the rollover test requirements prior to bus manufacturing

Results: From the simulation of the rollover test of the superstructure section of the Miral Infiniti interprovincial bus in the initial Hino Ak chassis, it resulted in the survival space being invaded by $20.78 \mathrm{~mm}$. When making the modification of the superstructure of: the height of the floor, occupant capacity, distance between seats and dimension and location of the

\footnotetext{
1 G+ Energy-Risks \& Engineering Group, Department of Food Science and Engineering, Technical University of Ambato, Ambato-Ecuador, ma.cordova@uta.edu.ec

2 Faculty of Mechanical Engineering, Technical University of Ambato, Ambato-Ecuador, ch.arroba@uta.edu.ec

3 Factulty of Animal Sciences, Chimborazo Polytechnic High School, Riobamba-Ecuador, cristian.santiana@espoch.edu.ec

4 Factulty of Animal Sciences, Chimborazo Polytechnic High School, Riobamba-Ecuador, gcarrera@espoch.edu.ec
} 
skylights, the second simulation determines: an impact speed of: $7.18 \mathrm{~m} / \mathrm{s}$; an angular impact velocity of $3.78 \mathrm{rad} / \mathrm{s}$; distance between the survival space and the superstructure with a maximum of $350 \mathrm{~mm}$ at the start of the test does not invade the survival space.

Conclution: With a modification of the superstructure and an expense of USD 3920, the superstructure with $4033 \mathrm{~kg}$ of total live load, an aerodynamic drag of 251,015 $\mathrm{kg}$, and a braking load of $4186 \mathrm{~kg}$ proposed for the Miral brand Infiniti model bus for Hino chassis Ak in the case of overturning resists without invading the survival space guaranteeing the safety of the occupants.

Keywords: Ansys, simulation, survival space, safety

\section{Resumen}

Se estudió el espacio de supervivencia en una sección de la superestructura del Autobús de servicio interprovincial fabricado por Miral Autobuses mediante el software, Ansys y Ls-Dyna sometido a un ensayo de vuelco. Primero se caracterizó el material que utiliza Miral Autobuses en la fabricación de la superestructura del autobús determinando propiedades mecánicas que son necesarios para el software Ls-Dyna. Se realizó una validación de resultados emitidos por Ls-Dyna simulando los ensayos de tracción, flexión, y flexión de una sección del pórtico y comparando con los resultados de ensayos físicos realizados en la maquina universal.

Métodos: El estudio inicia con la identificación de los materiales requeridos por el fabricante según la normativa Norma técnica ecuatoriana NTE INEN 1323:2009 primera revisión "Vehículos Automotores. Carrocerías de Buses Requisitos" y luego se efectuó la simulación de vuelco con el software Ansys y Ls-Dyna de una sección de la superestructura del autobús para determinar si el espacio de supervivencia es invadido, encontrando oportunidades de mejora y posteriormente realizar un rediseño de la superestructura y cumplir el Reglamento $\mathrm{N}^{\circ} 66$ de la Comisión Económica de las Naciones Unidas para Europa para los requerimientos del ensayo de vuelco previos a la fabricación del autobús.

Resultados: De la simulación del ensayo de vuelco de la sección de la superestructura del autobús interprovincial marca Miral Infiniti en chasis Hino Ak inicial dio como resultado que el espacio de supervivencia es invadido en $20.78 \mathrm{~mm}$. Al realizar la modificación de la superestructura de: la altura del piso, capacidad de ocupantes, distancia entre asientos y dimensión y ubicación de las claraboyas, la segunda simulación determina: una velocidad de impacto de: $7,18 \mathrm{~m} / \mathrm{s}$; una velocidad angular de impacto de 3,78 rad/s; distancia entre el espacio de supervivencia y la superestructura con un máximo de $350 \mathrm{~mm}$ al inicio del ensayo no invade el espacio de supervivencia.

Conclusión: Con una modificación de la superestructura y un gasto de USD 3920, la superestructura con $4033 \mathrm{~kg}$ de carga total en vivo, una resistencia aerodinámica de $251,015 \mathrm{~kg}$ y una carga de frenado de $4186 \mathrm{~kg}$ propuesta para el modelo de bus Infiniti de la marca Miral para el chasis Hino Ak en el caso de un vuelco resiste sin invadir el espacio de supervivencia garantizando la seguridad de los ocupantes.

Palabras Claves: Ansys , simulación, espacio de supervivencia, seguridad 


\section{Introducción.}

Las actividades de transporte de personas determinan el uso de autobuses interprovinciales que comúnmente sufren accidentes de tránsito con consecuencias catastróficas para los usuarios (Robert Sentís, 2016). Es por esto que las normativas de construcción de las estructuras de los autobuses incluyen aparte del cumplimiento de las normas de materiales el cumplimiento de ensayos de simulación para poder homologar las estructuras antes de salir al mercado y que son controlados por organismos competentes a nivel nacional e internacional(Mayanza Balla y Tuquinga Sagñay, 2018). Uno de esos ensayos es el ensayo de vuelco (Alcalá Fazio, 1997).

En los países de la Comunidad Europea, fue España uno de los primeros países en establecer la obligatoriedad del ensayo de vuelco (Gómez, 2009) en la actualidad Ecuador también ha visto la necesidad de realizar esta prueba en nuestro país. El ensayo de vuelco consiste en someter una volcadura del autobús en condiciones controladas para esto no es necesario que el autobús no esté totalmente terminado, será representativo de la producción de vehículos en cuanto a su masa en vacío en orden de marcha, centro de gravedad y distribución de masas. El área de impacto será de cemento u otro material rígido (Donaire, 2016).(López Ortiz, 2016)

Actualmente está apareciendo reglamentación que impone requisitos a las estructuras y sistemas de retención de los autobuses y autocares para garantizar la seguridad de sus ocupantes. Este hecho está marcando de forma importante la evolución de la seguridad pasiva de este tipo de vehículos. La reglamentación Europea está consiguiendo que en los últimos años se hayan realizado numerosos avances en seguridad que han conllevado la necesidad de estudios de investigación para determinar los requisitos a imponer sobre los mismos (Gonzalez, 2010).

El acondicionamiento del habitáculo resulta vital para garantizar la seguridad de los ocupantes. Entre las medidas técnicas que suelen utilizar los fabricantes para aumentar la capacidad de seguridad pasiva del habitáculo pueden citarse: la concepción estructural que permita un amplio margen de supervivencia (Gomez y Navarro, 2011), el equipamiento de los sistemas apropiados para la sujeción de los ocupantes, la funcionalidad plena después del accidente, de los elementos y conjuntos para la liberación de los ocupantes, la protección contra incendios, el sistema de fijación del parabrisas y la Anti-intrusión de piezas de la carrocería por el parabrisas y luna trasera.

El espacio de supervivencia debe quedar en el compartimento de viajeros mientras se somete la superestructura a uno de los ensayos de vuelco y después de dicho ensayo, también se denomina el volumen que se obtienen en el compartimento de ocupantes desplazando en línea recta el plano vertical y transversal. El espacio de supervivencia entre el plano más atrasado y el más adelantado del compartimento o compartimentos de viajeros, del personal y del conductor es continuo y se determina desplazando, por toda la longitud del vehículo, a lo largo de líneas rectas y a través de los puntos de Para simular el peor caso en un grupo de tipos de vehículo y permitir futuros avances de diseño, el fabricante podrá definir un espacio de supervivencia mayor de lo necesario para una disposición de asiento determinada(Liu et al., 2018).

Este trabajo contempla la determinación de los factores preponderantes de la superestructura, su mejora y la verificación de la invasión de la estructura mejorada en el área de supervivencia utilizando 
el software Ansys y Ls-Dyna (Ding et al., 2012).

\section{MATERIALES Y MÉTODO}

\section{Comportamiento mecánico}

El estudio del comportamiento mecánico de una sección de la superestructura del autobús interprovincial se realizó siguiendo el diagrama de flujo del esquema 1.

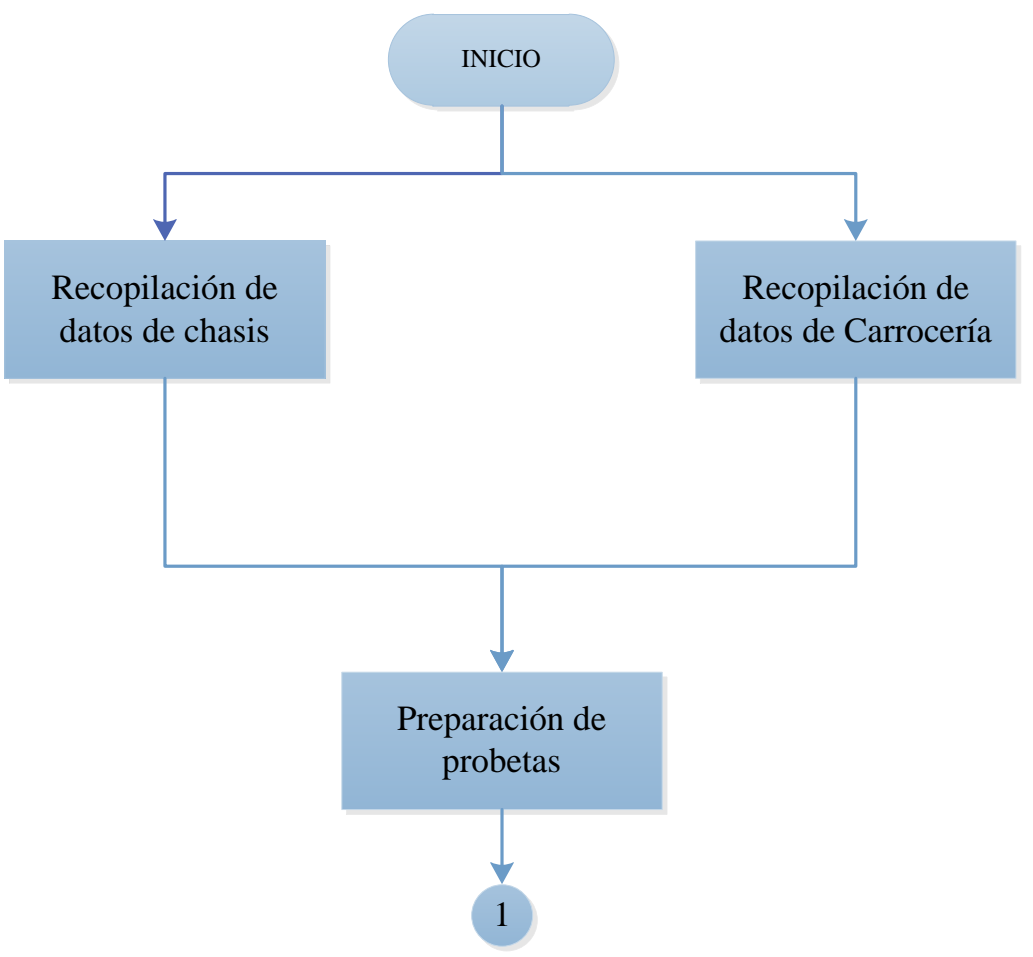



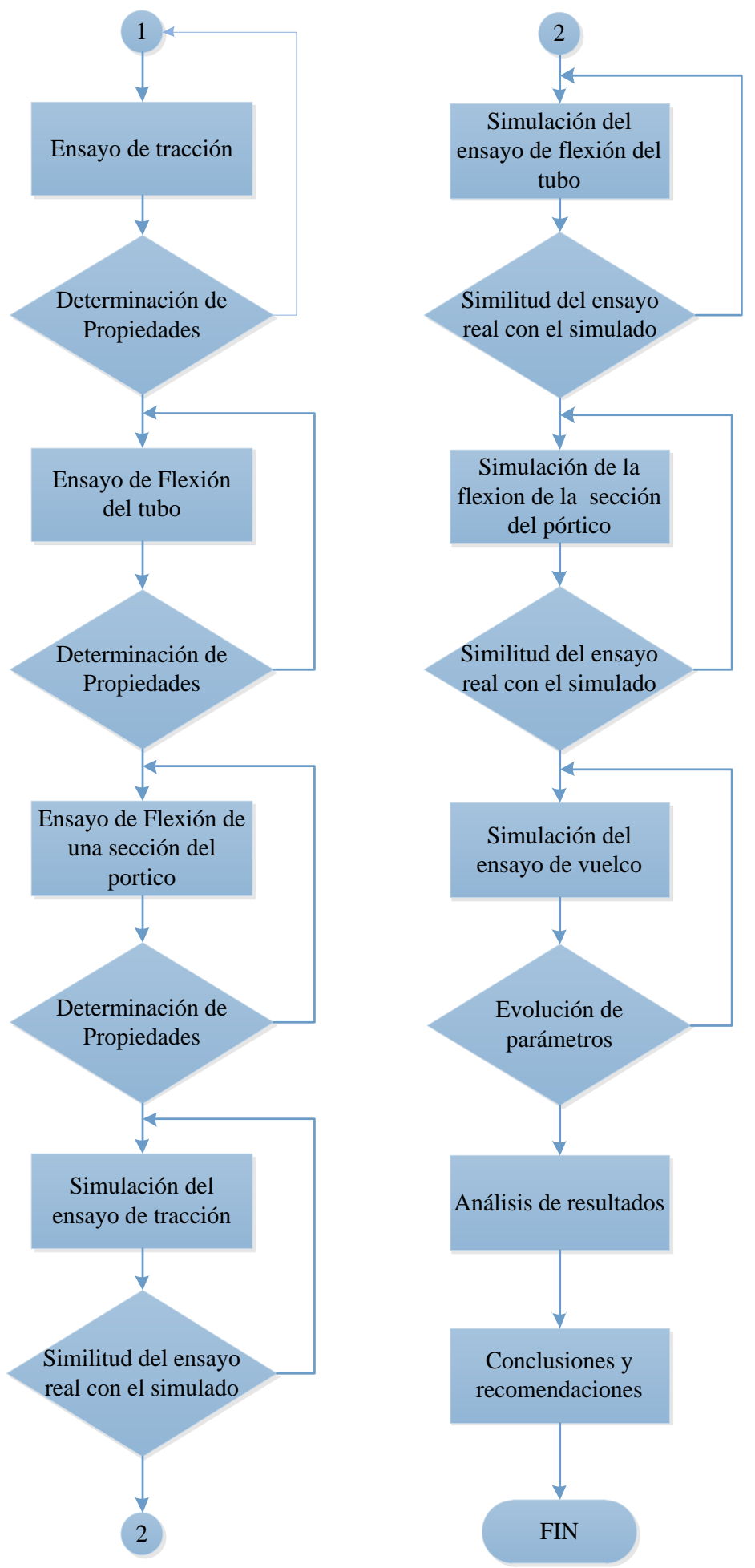

Figura 1. Estudio del comportamiento mecánico de un autobús interprovincial 


\section{Materiales de la superestructura}

La superestructura del autobús está constituida de varios elementos, de diferente geometría, y sección destinada a soportar las diferentes cargas que actúan sobre el autobús en su normal funcionamiento y en caso de un accidente (López Ortiz, 2016)

\section{Capacidad de carga del autobús}

La capacidad de carga se determinó según la norma INEN 1323, 2009 ANEXO B. Según la regulación nacional de transporte de pasajeros para el servicio interprovincial no es permitido transportar pasajero de pie y se considera el peso estimado por pasajero y el equipaje de mano (Andrade García et al., 2012).

\section{Determinación de la distribución de masas en puntos de apoyo del autobús}

La distribución de pesos en vacío que es soportado en cada uno de los neumáticos que se consideran puntos de apoyo del autobús fabricado en chasis marca Hino Ak y con carrocería Modelo Infiniti de la cooperativa Santo Domingo, se realizó en las instalaciones de BIOALIMENTAR en la balanza que se muestra utilizada para la recepción y despacho de productos destinados a la alimentación de animales domésticos.

\section{Calculo de la ubicación del centro de gravedad del autobús}

La posición del centro de gravedad del vehículo se determinará en condición de masa en orden de marcha(Reglamento_n ${ }^{\circ}$ 66_(CEPE/ONU), 2007). Se entenderá por masa en orden de marcha la masa del vehículo, sin ocupantes ni carga, la masa del combustible correspondiente al $90 \%$ de la capacidad del depósito especificada por el fabricante y en su caso, la masa del refrigerante, el lubricante, las herramientas y la rueda de repuesto.

\section{Posición longitudinal del centro de gravedad}

La posición longitudinal (11) del centro de gravedad se determina con la siguiente ecuación:

$$
\left.l 1=\frac{(P 3+P 4) * L 1}{P_{\text {total }}} \quad \text { (EC. } 1\right)
$$

Donde:

11 = posición longitudinal del centro de gravedad.

$\mathrm{L} 1=$ distancia entre ejes del autobús.

$11=$ es la posición del centro de gravedad en la posición longitudinal.

\section{Posición transversal del centro de gravedad del autobús}

La posición transversal ( $\mathrm{t}$ ) del centro de gravedad del vehículo en relación con su plano central longitudinal vertical

Se determina con la siguiente ecuación:

Donde:

$$
\left.t=\left((P 1-P 2) \frac{T 1}{2}+(P 3-P 4) \frac{T 2}{2}\right) * \frac{1}{P_{\text {total }}} \quad \text { (EC. } 2\right)
$$

$\mathrm{t}=$ posición transversal del centro de gravedad 
$\mathrm{T} 1$ = la distancia entre los centros de la rueda a cada extremo del primer eje.

$\mathrm{T} 2$ = la distancia entre los centros de la rueda a cada extremo del segundo eje.

\section{Altura del transversal del centro de gravedad}

La altura del centro de gravedad (h0) se determinará basculando el vehículo en sentido longitudinal y midiendo la carga en los dos ejes. El ángulo de inclinación del ensayo de basculamiento se determinará mediante la ecuación 3.

$$
\alpha=\operatorname{arsin}\left(\frac{H}{L 1}\right)
$$

Donde:

$\mathrm{H}=$ la diferencia de altura entre los dibujos de las ruedas del primer y segundo eje.

$\alpha=$ Angulo formado entre el plano horizontal y la línea horizontal que se forma entre el eje delantero y posterior.

Verificamos la masa del vehículo de acuerdo a la ecuación 4.

$$
F_{\text {total }}=F 1+F 2+F 3+F 4 \quad \text { (EC. 4.) }
$$

Donde:

$\mathrm{F} 1$ = reacción de la carga bajo la rueda izquierda del primer eje.

F2 = reacción de la carga bajo la rueda derecha del primer eje.

F3 = reacción de la carga bajo las ruedas izquierdas del segundo eje.

F4 = reacción de la carga bajo las ruedas derechas del segundo eje.

$F_{\text {total }}=$ Peso total del autobús $=$ Ptotal.

La altura del centro de gravedad del vehículo viene dada por:

$$
h 0=r+\left(\frac{1}{\operatorname{tg} \alpha}\right)\left(l 1-L 1 \frac{F 3+F 4}{\text { Ptotal }}\right)
$$

Donde:

$r=$ la altura del centro de la rueda en el eje delantero por encima de la superficie de la balanza.

\section{Delimitación del espacio de supervivencia}

El espacio de supervivencia se determinó acuerdo con el Reglamento n66 CEPE/ONU, Homologación de vehículos de gran capacidad para el transporte de personas respecto a la resistencia mecánica de su superestructura, define el perímetro del espacio de supervivencia del vehículo se determinará creando en el interior del autobús. El punto de referencia (Sr) está situado en el respaldo de cada asiento exterior orientado hacia adelante o hacia atrás y a $150 \mathrm{~mm}$ de la superficie interior de la pared lateral.

\section{Simulación del ensayo de vuelco de la sección de la estructura}

El análisis se inicia dibujando la geometría de la sección de la superestructura tomando en cuento todos los elementos que contribuyen en la resistencia de la superestructura.

Para determinar el centro de gravedad de la sección de la superestructura se utiliza un software CAD. El ensayo en el banco se inicia se realizara desde cuando la sección de la superestructura comienza a impactar con el piso. El mallado y la calidad de la malla mostrada en la 
Figura 4.

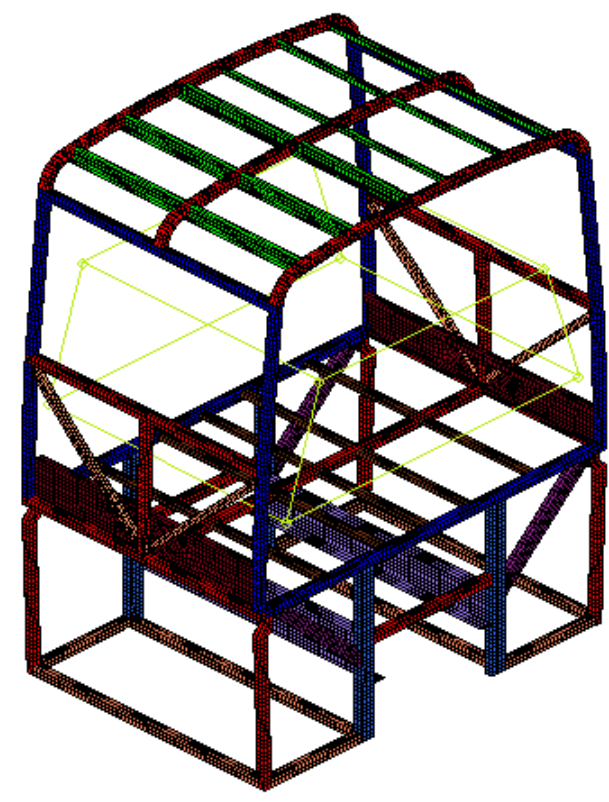

Figura 4. Malla de la sección de la estructura (Fuente: El Autor)

\section{RESULTADOS}

\section{Condiciones de la superestructura}

Se determinó los siguientes parámetros de la superestructura luego de los cálculos correspondientes utilizando las ecuaciones descritas.

Tabla 1. Condiciones superestructura

\begin{tabular}{|ll|}
\hline Parámetro & Valor \\
\hline Peso total $(\mathrm{kg})$ & 1100,00 \\
Posición longitudinal del centro de gravedad $(\mathrm{mm})$ & 2990,00 \\
Posición transversal del centro de gravedad $(\mathrm{mm})$ & 37,56 \\
Altura del transversal del centro de gravedad $(\mathrm{mm})$ & 200,00 \\
Carga uniformemente distribuida $(\mathrm{kg} / \mathrm{mm})$ & 0,60 \\
Mataerial superestructura & Acero estructural ASTM A 500 \\
\hline
\end{tabular}

Simulación con Solver de Ls-Dyna de vuelco de una sección de la superestructura Los resultados de la simulación de vuelco se observan en la figura 5. 
Vol. 3, N4.2, p. 86-99, diciembre, 2019

\begin{tabular}{|c|c|c|}
\hline $\mathrm{t}=0.0000 \mathrm{~s}$ & $t=0.0089 \mathrm{~s}$ & $\mathrm{t}=0.017 \mathrm{~s}$ \\
\hline $\mathrm{t}=0.026 \mathrm{~s}$ & $\mathrm{t}=0.035 \mathrm{~s}$ & $t=0.044 s$ \\
\hline $\mathrm{t}=0.054 \mathrm{~s}$ & $t=0.063 \mathrm{~s}$ & $\mathrm{t}=0.071 \mathrm{~s}$ \\
\hline $\mathrm{t}=0.081 \mathrm{~s}$ & $\mathrm{t}=0.089 \mathrm{~s}$ & $t=0.099 \mathrm{~s}$ \\
\hline$t=0.108$ & $\mathrm{t}=0.117 \mathrm{~s}$ & $\mathrm{t}=0.126 \mathrm{~s}$ \\
\hline $\mathrm{t}=0.135 \mathrm{~s}$ & $\mathrm{t}=0.144 \mathrm{~s}$ & $\mathrm{t}=0.153 \mathrm{~s}$ \\
\hline
\end{tabular}


Vol. 3, N4.2, p. 86-99, diciembre, 2019

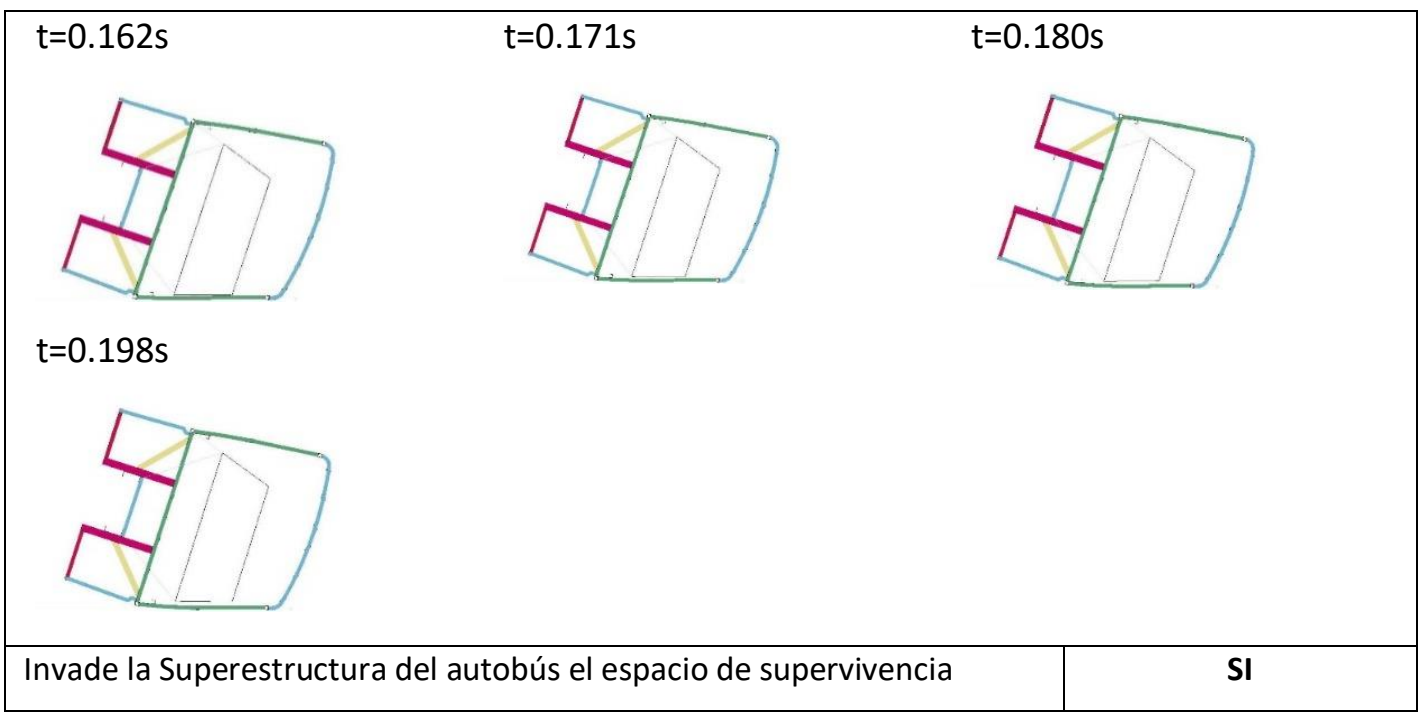

Figura 5. Resultados de simulación condiciones iniciales

Según el Solver de Ls-Dyna para el ensayo de vuelco de una sección dela superestructura, muestra la distancia que existe entre el espacio de supervivencia y la superestructura con un maximo de $350 \mathrm{~mm}$ al inicio del ensayo y luego es invadido por la superestrcutura en $20.78 \mathrm{~mm}$ cuando la superestuctura impacta al piso y sufre su mayor deformación, invadiendo el espacio de supervivencia.

\section{Simulación con Solver de Ls-Dyna de vuelco de una sección de la superestructura modificada}

Luego de modificar la secciones de color verde que se muestra en la figura 5. Se realizó la nueva simulación determinando los valores de la figura 6. Se consideró una estructura con las siguientes características generales de fabricación. La estructura de piso fabricado en tubería estructural cuadrado de 50x50x3mm correspondiente a los tubos de que conectan el anclaje del chasis con el piso de la carrocería, tubos cuadrados de 50x50X2 $\mathrm{mm}$ que se utilizan en la parte intermedia del piso entre los tubos de 50x50x3mm y canal u de 50x25x2mm correspondiente al tejido del piso.

Las estructuras de laterales construidas en tubo cuadrado de 50x50x3mm y reticulado con tubo de 50x50x2mm y tubo de 40x40x2 $\mathrm{mm}$. El techo en perfil cuadrado de 50x50x2mm, con y reticulado con perfil Z. La estructura del frente y respaldo en tubería estructural cuadrada de 40x40x2mm $50 \times 50 \times 2 \mathrm{~mm}, 25 \times 25 \times 2 \mathrm{~mm}$, elementos de refuerzo en perfiles de acero de $2 \mathrm{~mm}$ de espesor. El anclaje de la superestructura al chasis por medio de un arreglo perfil L 80x70x6mm y doble tubo 100x50x3mm, cada conexión con pernos de 1/2 in de diámetro grado 8 .

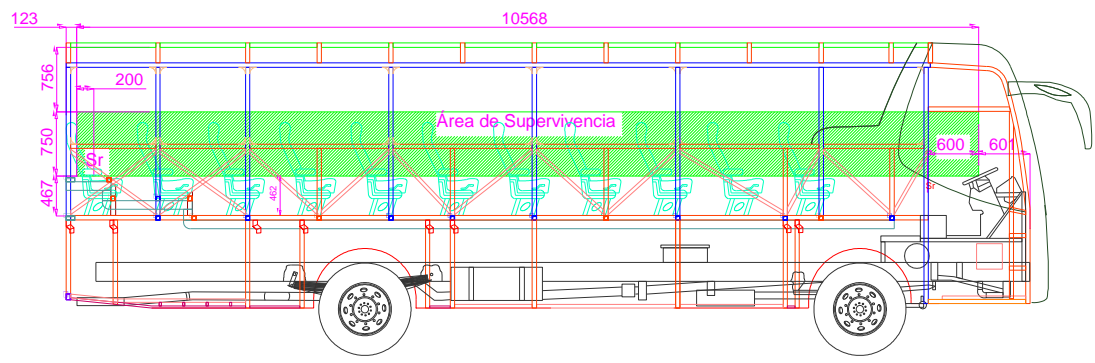

Figura 6. Área de supervivencia y secciones del bus quepueden ser modificadas. (Fuente: El Autor) 


\begin{tabular}{|c|c|c|}
\hline \multicolumn{3}{|c|}{ RESULTADOS DEL ENSAYO } \\
\hline$t=0.0000 s$ & $\mathrm{t}=0.0089 \mathrm{~s}$ & $t=0.017 \mathrm{~s}$ \\
\hline$t=0.026 s$ & $\mathrm{t}=0.035 \mathrm{~s}$ & $\mathrm{t}=0.044 \mathrm{~s}$ \\
\hline$t=0.054 \mathrm{~s}$ & $t=0.063 \mathrm{~s}$ & $t=0.071 \mathrm{~s}$ \\
\hline$t=0.081 \mathrm{~s}$ & $t=0.089 s$ & $t=0.099 \mathrm{~s}$ \\
\hline$t=0.108$ & $\mathrm{t}=0.117 \mathrm{~s}$ & $t=0.126 s$ \\
\hline$t=0.135 \mathrm{~s}$ & $t=0.144 s$ & $t=0.153 \mathrm{~s}$ \\
\hline
\end{tabular}




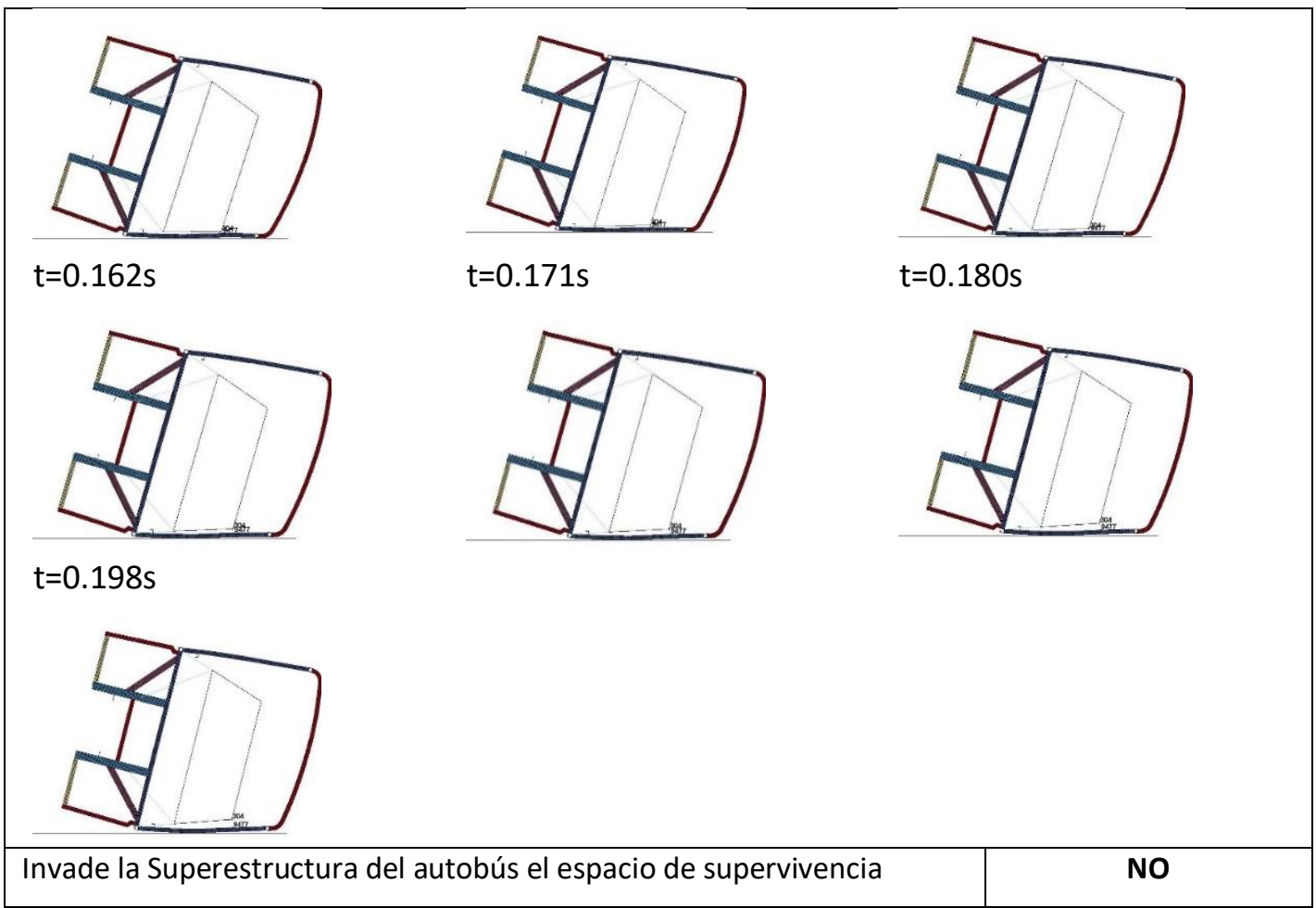

\section{Discusión.}

La simulación requiere mucho tiempo y de una computadora con un procesador sofisticado para resultados rápidos.

\section{Conclusiones}

- La modificación que se realizó en la superestructura ha mejorado el comportamiento de la misma cumpliendo de manera satisfactoria el ensayo de vuelco, aumentado la seguridad del autobús. Los resultados muestran la distancia que existe entre el espacio de supervivencia y la superestructura con un máximo de $350 \mathrm{~mm}$ al inicio del ensayo y un mínimo de $20.61 \mathrm{~mm}$ cuando la superestructura impacta al piso y sufre su mayor deformación, sin invadir el espacio de supervivencia. EL costo total de la implementación determina un valor de 3920 USD.

\section{Referencias Bibliográficas}

Alcalá Fazio, E. (1997). Optimización de las superestructuras de autobuses y autocares sometidos al vuelco lateral. Industriales.

Andrade García, L. E., Canchig, M. y Xavier, P. (2012). Estudio del diseño de una carrocería con análisis de esfuerzos estáticos y dinámicos de un autobús de la marca Patricio Cepeda calificada con norma ISO 9001-2008 mediante la aplicación de un software para el cálculo de las fuerzas. LATACUNGA/ESPE/2012. 
Ding, F., Bian-you, T., Yong-xin, Y., Li, W. y Hong, Z. (2012). Study on shock of toothed electromagnetic clutch based on ANSYS/LS-DYNA software [J]. Journal of Machine Design, 2.

Donaire, J. (2016). Comparativa de modelos de simulación de vuelco de autobuses segun los procedimientos del reglamento 66. España: Universidad Politécnica de Madrid.

Gómez, S. (2009). Simulación De Un Ensayo De Impacto Con Péndulo Sobre Un Autobús Mediante Ls-Dyna. Universidad Carlos III

Gomez, T. y Navarro, J. (2011). Estructura del Vehículo (Seguda Edicion ed.): Paraninfo.

Gonzalez, P. (2010). Modelacion y Analisis de la Superestrcutura de un Autobus y Diapress Mediante Elementos Finitos. Carlos III

Liu, L., Fang, C., Yang, J., Zhang, H., Huang, Y., Xuan, C., Wang, Y., Li, S., Sha, J., Zha, M. y Guo, M. (2018). The effect of noise exposure on insulin sensitivity in mice may be mediated by the JNK/IRS1 pathway. Environmental Health and Preventive Medicine, 23(1). doi: 10.1186/s12199-018-0694-3

López Ortiz, S. A. (2016). Determinación de configuraciones estructurales de un frente de autobús interprovincial y su incidencia en la resistencia al impacto frontal mediante el método de elementos finitos. Universidad Técnica de Ambato. Facultad de Ingeniería Civil y Mecánica ....

Mayanza Balla, J. A. y Tuquinga Sagñay, G. P. (2018). Análisis del comportamiento termomecánico de la junta soldada tipo $k$, aplicada en la fabricación de carrocería de autobuses interprovinciales. Escuela Superior Politécnica de Chimborazo.

Reglamento_n_66_(CEPE/ONU). (2007). Prescripciones técnicas uniformes relativas a la homologación de vehículos de grandes dimensiones para el transporte de pasajeros por lo que respecta a la resistencia de su superestructura Reglamento $n^{\circ} 66$ de la Comisión Económica de las Naciones Unidas para Europa (CEPE/ONU).

Robert Sentís, L. (2016). Salud laboral en conductores profesionales del transporte por carretera. Universitat Rovira i Virgili.

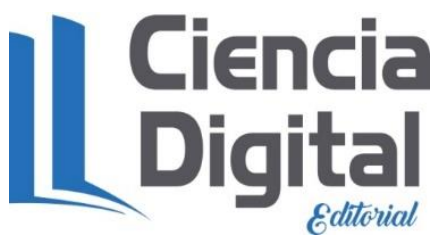


PARA CITAR EL ARTÍCULO INDEXADO.

Córdova M, Mera C. (2018). Estudio de factibilidad para la implementación de un laboratorio de análisis de lixiviados de la Empresa Pública Municipal Gestión Integral de Desechos Sólidos de Ambato (EPM- GIDSA).

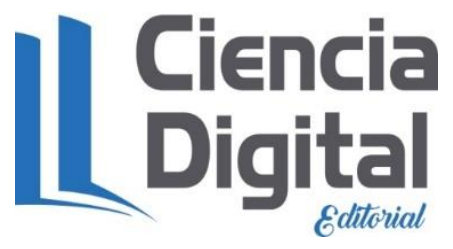

El artículo que se publica es de exclusiva responsabilidad de los autores y no necesariamente reflejan el pensamiento de la Revista Ciencia Digital.

El articulo queda en propiedad de la revista y, por tanto, su publicación parcial y/o total en otro medio tiene que ser autorizado por el director de la Revista Ciencia Digital.
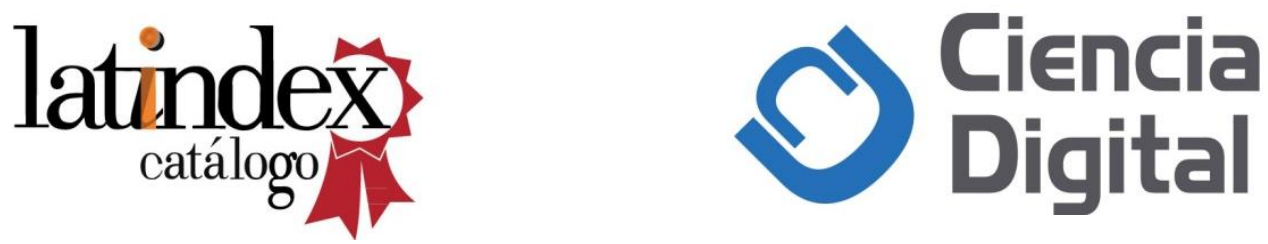